\title{
Newton-based Extremum Seeking for Higher Derivatives of Unknown Maps with Delays
}

\author{
Damir Rušiti ${ }^{1}$, Tiago Roux Oliveira ${ }^{2}$, Greg Mills $^{3}$, and Miroslav Krstic ${ }^{4}$
}

\begin{abstract}
We present a Newton-based extremum seeking algorithm for maximizing higher derivatives of unknown maps in the presence of time delays. Different from previous works about extremum seeking for higher derivatives, arbitrarily long input-output delays are allowed. We incorporate a predictor feedback with a perturbation-based estimate for the Hessian's inverse using a differential Riccati equation. As a bonus, the convergence rate of the real-time optimizer can be made user-assignable, rather than being dependent on the unknown Hessian of the higher-derivative map. Furthermore, exponential stability and convergence to a small neighborhood of the unknown extremum point can be obtained for locally quadratic derivatives by using backstepping transformation and averaging theory in infinite dimensions. We also give a numerical example in order to highlight the effectiveness of the proposed predictorbased extremum seeking for time-delay compensation.
\end{abstract}

\section{INTRODUCTION}

Time delays are some of the most common phenomena that arise in engineering practice and need to be handled carefully. In this paper, we analyze the problem of non-model based extremum seeking (ES) of higher derivatives in the presence of time delays. We consider scalar Newton-based ES under input-output delays. In recent years, there have been a lot of publications on ES in theory [22], [23], [24], [14], [15], [16], [17], [19] as well as applications [20], [21]. In [7] and [11] Newton-based ES in absence of delay was deeply studied. A highlight of these works is the approach used to estimate the Hessian's inverse of the nonlinear map, which is generated by means of Riccati filter. This is applied to remove the dependence of the algorithm's convergence rate on the second derivative (Hessian), making it userassignable. Mentioned works just handled the extremum seeking for the map itself.

However, there are applications were an extremum of the map's higher derivative is in focus. In [5] the authors represent a refrigeration system where a suitable operating point is located at the maximum negative slope which is subject to change. This point of zero curvature corresponds to a minimum of the first derivative of the input-output map.

The first author thanks to TUM, UCSD and UERJ for support.

${ }^{1}$ D. Rušiti is with Dept. of Information-oriented Control, Technical University of Munich (TUM), Munich, Germany. E-mail: damir.rusiti@tum.de

${ }^{2}$ T. R. Oliveira is with Dept. of Electronics and Telecommunication Engineering, State University of Rio de Janeiro (UERJ), Rio de Janeiro, RJ 20550-900, Brazil. E-mail: tiagoroux@uerj.br

${ }^{3} \mathrm{G}$. Mills is with Dept. of Mechanical and Aerospace Engineering, University of California - San Diego (UCSD), La Jolla, CA 92093-0411, USA. E-mail: glmillseucsd.edu

${ }^{4} \mathrm{M}$. Krstić is with Dept. of Mechanical and Aerospace Engineering, University of California - San Diego (UCSD), La Jolla, CA 92093-0411, USA. E-mail: krstic@ucsd.edu
Hence, being able to track the minimum of the first derivative in real-time would allow the system to operate almost whole time in the most suitable operating condition.

A Newton-based ES generalization was presented in [12] to maximize arbitrary higher derivatives of an unknown map. Using sinusoidal perturbations, estimation of the gradient and the Hessian of map's $n^{\text {th }}$ derivative were obtained as well as the local stability proof for the closed-loop system. However, reference [12] does not handle the time delay case. On the other hand, the first works which deal with Newton-based ES in the presence of constant and known time delays are references [1], [2], [3]. Unfortunately, the authors of [1], [2], [3] just handled the extremum seeking for the map itself.

In this paper, we extend the applicability and usage of the predictor-based controller with an averaging-based estimate of the Hessian's inverse proposed in [3] to maximize higher derivatives of a static map despite the presence of time delays.

We rigorously prove the stability for locally quadratic derivatives via backstepping transformation [8] and averaging theory in infinite dimensions [9], [10], considering the whole system which is infinite dimensional due to delays. Simulation results show the applicability of the proposed algorithm.

Notation and Norms: The 2-norm of a finitedimensional (ODE) state vector $X(t)$ is denoted by single bars, $|X(t)|$. In contrast, norms of functions (of $x$ ) are denoted by double bars. By default, $\|\cdot\|$ denotes the spatial $L_{2}[0, D]$ norm, i.e., $\|\cdot\|=\|\cdot\|_{L_{2}[0, D]}$. Since the PDE state variable $u(x, t)$ is a function of two arguments, we should emphasize that taking a norm in one of the variables makes the norm a function of the other variable. For example, the $L_{2}[0, D]$ norm of $u(x, t)$ in $x \in[0, D]$ is $\|u(t)\|=\left(\int_{0}^{D} u^{2}(x, t) d x\right)^{1 / 2}$. The partial derivatives of $u(x, t)$ are denoted by $u_{t}(x, t)$ and $u_{x}(x, t)$ or, occasionally, by $\partial_{t} u_{\text {av }}(x, t)$ and $\partial_{x} u_{\text {av }}(x, t)$ to refere the operator for its average signal $u_{\text {av }}(x, t)$.

\section{Newton-Based Extremum Seeking OF Higher DERIVATIVES FOR DELAY SYSTEMS}

Scalar ES considers applications in which one want to maximize (or minimize) the output $y \in \mathbb{R}$ of an unknown nonlinear static map $h(\theta)$ by varying the input $\theta \in \mathbb{R}$ in real time. But like in many technical applications we have to consider that the output may be time-delayed and hence, we additionally assume that there is a constant and known 


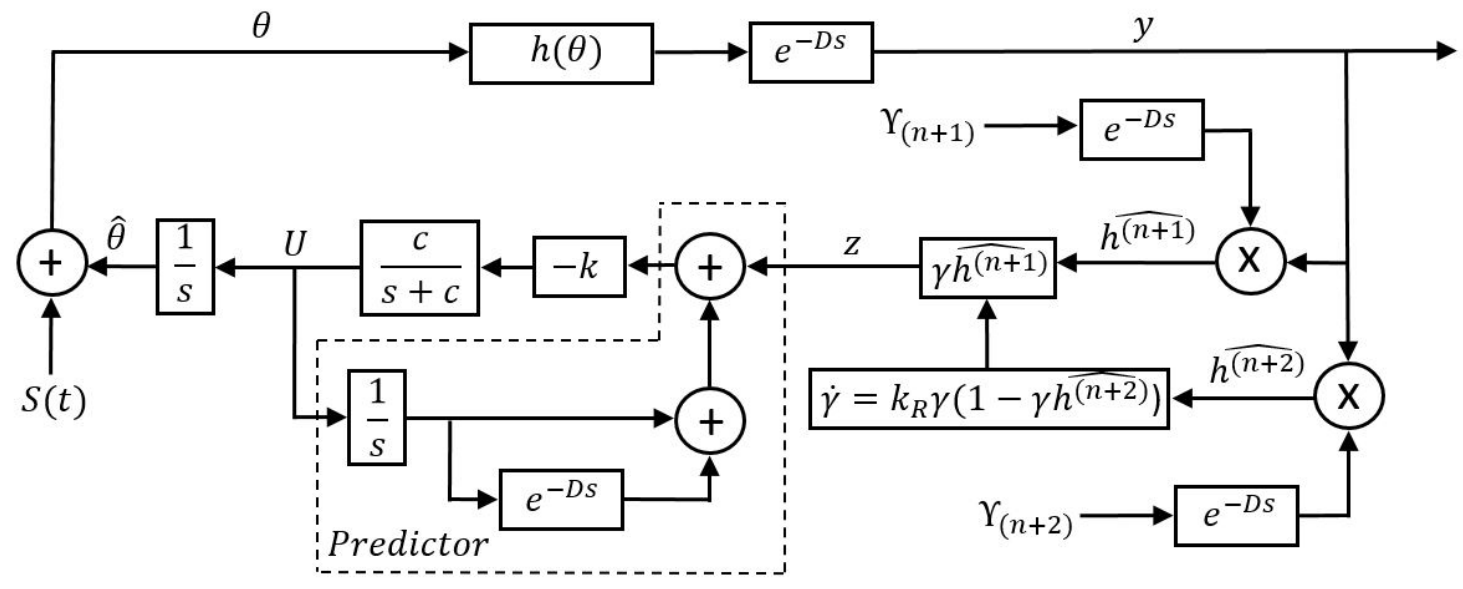

Fig. 1. Block diagram of the basic prediction scheme for output-delay compensation in Newton-based ES for maximizing higher derivatives. The predictor feedback with a perturbation-based estimate of the Hessian's inverse obeys equations (9), (6) and (30). The dither and demodulating signals are given by $S(t), \Upsilon_{(n+1)}$ and $\Upsilon_{(n+2)}$ which are calculated according to (7b)-(7d) and (8).

delay $D \geq 0$ such that the output is expressed by

$$
y(t)=h(\theta(t-D)) .
$$

As done in [3], in this work we also assume that our system is output-delayed. In Fig. 1, we illustrate the proposed scalar version of the Newton-based ES for maximization of higher derivatives based on predictor feedback for delay compensation.

Without loss of generality, let us consider the maximization of $n^{t h}$ derivative of the output in the presence of time delay using Newton-based ES, where the maximizing value of $\theta$ is denoted by $\theta^{*}$. We state our optimization problem as follows

$$
\max _{\hat{\theta} \in \mathbb{R}} h^{(n)}(\hat{\theta}) .
$$

Assumption 1: Let $h^{(n)}(\cdot)$ be the $n^{\text {th }}$ derivative of a smooth function $h(\cdot): \mathbb{R} \rightarrow \mathbb{R}$. Now let us define

$$
\theta_{\max }=\left\{\theta \mid h^{(n+1)}(\theta)=0, \quad h^{(n+2)}(\theta)<0\right\}
$$

to be a collection of maxima where $h^{(n)}$ is locally concave. Now assume that $\theta^{*} \in \theta_{\max }$ and $\theta_{\max } \neq \emptyset$. For simplicity and without loss of generality, we assume that $\theta_{\max }=0$. The design parameters $k, k_{R}, a, \omega, c,>0$ are presented in Fig. 1. According to [12], we switch from maximization to minimization problem by setting $\operatorname{sgn}\left(\gamma_{0}\right)=\operatorname{sgn}\left(h^{(n+2)}(0)\right)$ with $\gamma_{0}$ as initial value of $\gamma$.

\section{A. System and Signals}

Let $\hat{\theta}$ be the estimate of maximum point and

$$
\tilde{\theta}(t)=\hat{\theta}(t)-\theta^{*}
$$

be the estimation error.

From the block diagram in Fig. 1, the error dynamics can be written as

$$
\dot{\tilde{\theta}}(t)=\dot{\hat{\theta}}(t)=U(t) .
$$

Moreover we have

$$
\dot{\gamma}=k_{R} \gamma\left(1-\gamma \widehat{h^{(n+2)}}\right)
$$

where (6) is a differential Riccati equation.

From [12], the demodulated signals, $\widehat{h^{(j)}}=y \Upsilon_{j}$, are used to estimate the gradient $(j=n+1)$ and the Hessian $(j=n+2)$ of $h^{(n)}$. The equation (6) will be used to generate an estimate of the Hessian's inverse.

Now, we rearrange the equations given in [12], by changing them to the following:

$$
\begin{gathered}
\theta=\hat{\theta}+a \sin (\omega t), \\
\Upsilon_{j}=C_{j} \sin \left(j \omega t+\frac{\pi}{4}(-1)^{n+1}\left(1+(-1)^{j}\right)\right), \\
C_{j}=\frac{2^{j} j !}{a^{j}}(-1)^{F}, \\
F=\frac{j-\left|\sin \left(\frac{j \pi}{2}\right)\right|}{2},
\end{gathered}
$$

where $j=n+1$ for the gradient and $j=n+2$ for the Hessian estimate. We define the additive dither signal as

$$
S(t)=a \sin (\omega t)
$$

which is not delayed. However, as shown in Fig. 1, the multiplicative dither signals $\Upsilon_{(n+1)}$ and $\Upsilon_{(n+2)}$ are delayed by $D$ in order to cope with the delayed output $y$ [1]. As discussed in [3], [2] one could also advance the dither signal (8) with D time units instead of delaying $\Upsilon_{(n+1)}$ and $\Upsilon_{(n+2)}$.

Furthermore, let us define the measurable signal

$$
z(t)=\gamma(t) \widehat{h^{(n+1)}}
$$

where $\gamma$ is updated according to (6) and $\widehat{h^{(n+1)}}$ being the demodulated signal. We can construct (9), because we only use measurable/available signals $\gamma(t)$ and $\widehat{h^{(n+1)}}$. 


\section{B. Averaging Analysis}

Without loss of generality, the authors in [12], used equation (4) with $\theta^{*}=0$ to write the following error transformation

$$
\begin{aligned}
& \tilde{\theta}=\hat{\theta}, \\
& \tilde{\gamma}=\gamma-\underbrace{\frac{1}{h^{(n+2)}(0)}}_{H^{-1}} .
\end{aligned}
$$

Consider the particular average version of the signal $\eta_{j}:=$ $\Upsilon_{j} y$ which is given by

$$
\eta_{j}\left(\tilde{\theta}_{\mathrm{av}}\right)=\frac{1}{\Pi} \int_{0}^{\Pi} \Upsilon_{j} h\left(\tilde{\theta}_{\mathrm{av}}+a \sin (\sigma)\right) d \sigma,
$$

where $\Pi=2 \pi / \omega$. Now, one can provide a convenient expression of the demodulated signal $\eta_{j}\left(\tilde{\theta}_{\mathrm{av}}\right)=\widehat{h^{(n+1)}}$ for arbitrary $j=n+1$ as follows

$$
\eta_{j}\left(\tilde{\theta}_{\mathrm{av}}\right)=h^{(j)}\left(\tilde{\theta}_{\mathrm{av}}\right)+\underbrace{\frac{h^{(j+2)}\left(\tilde{\theta}_{\mathrm{av}}\right)}{4(j+1)} a^{2}+\mathcal{O}\left(a^{4}\right)}_{=X} .
$$

Futhermore, according to [12], the solutions of (10) and (11), when changing to faster time scale $\tau=\omega t$, are

$$
\begin{aligned}
& \tilde{\theta}_{\mathrm{avE}}=\tilde{\theta}^{*}+\mathcal{O}\left(a^{3}\right), \\
& \tilde{\gamma}_{\mathrm{avE}}=\tilde{\gamma}^{*}+\mathcal{O}\left(a^{3}\right),
\end{aligned}
$$

where

$$
\begin{aligned}
\tilde{\theta}^{*} & =\frac{h^{(n+3)}(0)}{4(n+2) h^{(n+2)}(0)} a^{2}, \\
\tilde{\gamma}^{*} & =\frac{\left(h^{(n+3)}(0)\right)^{2}}{4(n+2)\left(h^{(n+2)}(0)\right)^{3}} a^{2} \\
& +\frac{h^{(n+4)}(0)}{4(n+3)\left(h^{(n+2)}(0)\right)^{2}} a^{2} .
\end{aligned}
$$

All maps satisfying Assumption 1 can be approximated locally by a quadratic function

$$
h^{(n)}(\theta)=Q^{*}+\frac{H}{2}\left(\theta-\theta^{*}\right)^{2},
$$

for some constants $Q^{*}>0$ and $H<0$, where $H$ is the Hessian of the quadratic approximation [13]. Noticing that $X=0$ for locally quadratic assumption of $h^{(n)}$, plugging (16) into (13) we get

$$
\eta_{n+1}\left(\tilde{\theta}_{\mathrm{av}}\right)(t)=H \tilde{\theta}_{\mathrm{av}}(t-D) .
$$

Analogously to [12], the following delayed equations for the average error system can be written

$$
\begin{aligned}
& \frac{d \tilde{\theta}_{\mathrm{av}}(t)}{d t}=-k \gamma_{\mathrm{av}} \eta_{n+1}\left(\tilde{\theta}_{\mathrm{av}}(t-D)\right), \\
& \frac{d \tilde{\gamma}_{\mathrm{av}}(t)}{d t}=k_{R} \gamma_{\mathrm{av}}\left(1-\gamma_{\mathrm{av}} \eta_{n+2}\left(\tilde{\theta}_{\mathrm{av}}(t-D)\right)\right),
\end{aligned}
$$

with $\gamma \neq 0$ and $\gamma_{\mathrm{av}} \neq 0$. Hence, the right-hand side of (18) is zero for $\eta_{n+1}\left(\tilde{\theta}_{\mathrm{av}}\right)=0$, and it follows from (17) that the equilibrium of (18) is $\tilde{\theta}_{\mathrm{av}}=0$. In order to obtain the equilibrium for (19), we first calculate $\eta_{n+2}\left(\tilde{\theta}_{\text {av }}\right)$ which is simply $\mathrm{H}$. It follows that (19) is zero for $\left(1-\gamma_{\mathrm{av}} H\right)=0$ which is true for $\gamma_{\mathrm{av}}=H^{-1}$. Now, consider the equation (9) which can be written in averaged version as

$$
z_{\mathrm{av}}(t)=\gamma_{\mathrm{av}}(t) \eta_{n+1}\left(\tilde{\theta}_{\mathrm{av}}(t)\right) .
$$

We linearize $z_{\text {av }}$ in (20) at $\gamma_{\text {av }}(t)=1 / h^{(n+2)}(0)=H^{-1}$ and $\tilde{\theta}_{\mathrm{av}}=0$, under Assumption 1, and get for (9) a convenient expression

$$
z_{\mathrm{av}}(t)=\tilde{\theta}_{\mathrm{av}}(t-D) .
$$

\section{Motivation for Predictor Feedback}

From (5) it follows

$$
\dot{\tilde{\theta}}(t-D)=U(t-D) .
$$

Now using (5), the following "shifted" average model can be derived

$$
\dot{\tilde{\theta}}_{\mathrm{av}}(t)=U_{\mathrm{av}}(t),
$$

with $U_{\text {av }} \in \mathbb{R}$ being the resulting average control for $U \in \mathbb{R}$. One can try to feed back the future state $z_{\mathrm{av}}(\sigma+D)$ in the equivalent average system where $\sigma$ is time. Assuming that $\sigma=t-D$ we get

$$
z_{\mathrm{av}}(\sigma+D)=z_{\mathrm{av}}(t-D+D)=z_{\mathrm{av}}(t)
$$

and thus time-delay compensated signal which we feed back.

The delayed version of (23) is $\dot{\tilde{\theta}}_{\mathrm{av}}(t-D)=U_{\text {av }}(t-D)$. Applying (21) and (23) one has

$$
\dot{z}_{\mathrm{av}}(t)=U_{\mathrm{av}}(t-D) .
$$

Feeding back the future state $z_{\mathrm{av}}(t+D)$ for the delay compensation motivates the use of predictor feedback design.

Applying variation of constants [8], the future state is being calculated as

$$
z_{\mathrm{av}}(t+D)=z_{a v}(t)+\int_{t-D}^{t} U_{\mathrm{av}}(\sigma) d \sigma .
$$

We derive a controller as follows

$$
U_{\mathrm{av}}(t)=\dot{\tilde{\theta}}_{\mathrm{av}}(t)=-k z_{\mathrm{av}}(t+D),
$$

$\forall t \geq D$ and $k>0$, which yields to an average predictorbased control

$$
U_{\mathrm{av}}(t)=-k\left[z_{\mathrm{av}}(t)+\int_{t-D}^{t} U_{\mathrm{av}}(\sigma) d \sigma\right] .
$$

Furthermore using (20), (27) as well as time shifting we obtain the average error dynamics of the following form

$$
\dot{\tilde{\theta}}_{\mathrm{av}}(t)=-k z_{\mathrm{av}}(t+D)=-k \tilde{\theta}_{\mathrm{av}}(t),
$$

$\forall t \geq D$, with eigenvalues which are determined by $-k$. Thus, the system has an exponentially attractive equilibrium. 


\section{Predictor Feedback}

We propose the predictor-based controller which incorporates low-pass filter [3]

$$
U(t)=\frac{c}{s+c}\left\{-k\left[z(t)+\int_{t-D}^{t} U(\tau) d \tau\right]\right\}
$$

where $c>0$ is sufficiently large, i.e., the predictor feedback is of the form of a low-pass filtered of the non average version of (28). Note that we mix the time and frequency domain notation in (30) by using the braces $\{\cdot\}$ to denote that the transfer function acts as an operator on a time-domain function. The difficulty arises in the application of existing Averaging Theorem to infinite dimensions due to delays [9], [10]. Hence, we employs a low-pass filter, to still achieve our control objectives by transforming the original distributed input-output delays into state-delays for analysis purposes.

\section{StABILITY ANALYSIS}

In the following we give the main stability/convergence result for the closed-loop system using steps stated in [3].

Theorem 1: There exists $c^{*}>0$ such that, $\forall c \geq c^{*}$, $\exists \omega^{*}(c)>0$ such that, $\forall \omega>\omega^{*}$, the delayed closed-loop system (22) and (30), with $z(t)$ in (9) $\widehat{h^{(n+1)}}$ calculated as in in (7), $\gamma(t)$ in (6) and state $\tilde{\gamma}(t), \tilde{\theta}(t-D), U(\sigma)$, $\forall \sigma \in[t-D, t]$, has a unique locally exponentially stable periodic solution in $t$ of period $\Pi=2 \pi / \omega$, denoted by $\tilde{\gamma}^{\Pi}(t)$, $\tilde{\theta}^{\Pi}(t-D), U^{\Pi}(\sigma), \forall \sigma \in[t-D, t]$, satisfying, $\forall t \geq 0$ :

$$
\begin{aligned}
& \left(\left|\tilde{\gamma}^{\Pi}(t)\right|^{2}+\left|\tilde{\theta}^{\Pi}(t-D)\right|^{2}\right. \\
& \left.\quad+\left[U^{\Pi}(t)\right]^{2}+\int_{t-D}^{t}\left[U^{\Pi}(\tau)\right]^{2} d \tau\right)^{1 / 2} \leq \mathcal{O}\left(\frac{1}{\omega}\right) .
\end{aligned}
$$

Furthermore,

$$
\limsup _{t \rightarrow+\infty}\left|\theta(t)-\theta^{*}\right|=\mathcal{O}(a+1 / \omega) .
$$

Proof: The demonstration follows steps A to $\mathrm{G}$.

\section{A. Transport PDE for Delay Representation}

According to [8], the delayed version of (5) can be represented using a transport PDE as

$$
\begin{aligned}
\dot{\tilde{\theta}}(t-D) & =u(0, t), \\
u_{t}(x, t) & =u_{x}(x, t), \quad x \in[0, D], \\
u(D, t) & =U(t),
\end{aligned}
$$

where the solution of (34)-(35) is

$$
u(x, t)=U(t+x-D) .
$$

\section{B. Equations of the Closed-loop System}

First we derive an expression for the output in terms of $\tilde{\theta}$ using (7a) and plugging it into (1)

$$
y(t)=h(\tilde{\theta}(t-D)+a \sin (\omega(t-D)) .
$$

By representing the integrand in (30) using transport PDE state it follows

$$
U(t)=\frac{c}{s+c}\left\{-k\left[z(t)+\int_{0}^{D} u(\sigma, t) d \sigma\right]\right\}
$$

with $z(t)$ as in (9). Now using (37) and (38) one can rewrite (33)-(34) as

$$
\begin{aligned}
\dot{\tilde{\theta}}(t-D) & =u(0, t) \\
\partial_{t} u(x, t) & =\partial_{x} u(x, t), \quad x \in[0, D] \\
u(D, t) & =\frac{c}{s+c}\left\{-k\left[z(t)+\int_{0}^{D} u(\sigma, t) d \sigma\right]\right\} .
\end{aligned}
$$

\section{Average Model of the Closed-loop System}

The average version of system (39)-(41) is:

$$
\begin{aligned}
\dot{\tilde{\theta}}_{\mathrm{av}}(t-D) & =u_{\mathrm{av}}(0, t) \\
\partial_{t} u_{\mathrm{av}}(x, t) & =\partial_{x} u_{\mathrm{av}}(x, t), \quad x \in[0, D] \\
u_{\mathrm{av}}(D, t) & =\frac{c}{s+c}\left\{-k\left[z_{\mathrm{av}}(t)+\int_{0}^{D} u_{\mathrm{av}}(\sigma, t) d \sigma\right]\right\} .
\end{aligned}
$$

Denoting

$$
\begin{aligned}
\tilde{\vartheta}(t) & =\tilde{\theta}(t-D), \\
\tilde{\vartheta}_{\mathrm{av}}(t) & =\tilde{\theta}_{\mathrm{av}}(t-D)=z_{\mathrm{av}}(t),
\end{aligned}
$$

which is valid only locally because the quadratic condition invoked to derive (21), one has the following linearized average version of system (39)-(41):

$$
\begin{aligned}
\dot{\vartheta}_{\mathrm{av}}(t) & =u_{\mathrm{av}}(0, t) \\
\partial_{t} u_{\mathrm{av}}(x, t) & =\partial_{x} u_{\mathrm{av}}(x, t), \quad x \in[0, D] \\
\frac{d}{d t} u_{\mathrm{av}}(D, t) & =-c u_{\mathrm{av}}(D, t)-c k\left[\vartheta_{\mathrm{av}}(t)+\int_{0}^{D} u_{\mathrm{av}}(\sigma, t) d \sigma\right]
\end{aligned}
$$

where the filter $c / s+c$ is also represented in the state-space form. The solution of the transport PDE (48)-(49) is given by $u_{\mathrm{av}}(x, t)=U_{\mathrm{av}}(t+x-D)$.

From (6) and (16) we know that a local average for the Hessian's inverse estimation error is $\frac{d \tilde{\gamma}_{\mathrm{av}}(t)}{d t}=-k_{r} \tilde{\gamma}_{\mathrm{av}}(t)-$ $k_{r} H \tilde{\gamma}_{\text {av }}^{2}(t)$ and its linearized version

$$
\frac{d \tilde{\gamma}_{\mathrm{av}}(t)}{d t}=-k_{r} \tilde{\gamma}_{\mathrm{av}}(t)
$$

which is stable since $k_{r}$ is positive.

\section{Backstepping transformation, its inverse and the target system}

Consider the infinite-dimensional backstepping transformation of the delay state

$$
w(x, t)=u_{\mathrm{av}}(x, t)+k\left[\vartheta_{\mathrm{av}}(t)+\int_{0}^{x} u_{\mathrm{av}}(\sigma, t) d \sigma\right]
$$

which maps the system (47)-(49) into the target system

$$
\begin{aligned}
\dot{\tilde{\vartheta}}_{\mathrm{av}}(t) & =-k \vartheta_{\mathrm{av}}(t)+w(0, t), \\
w_{t}(x, t) & =w_{x}(x, t), \quad x \in[0, D], \\
w(D, t) & =-\frac{1}{c} \partial_{t} u_{\mathrm{av}}(D, t) .
\end{aligned}
$$


Using (51) for $x=D$ and the fact that $u_{\mathrm{av}}(D, t)=U_{\mathrm{av}}(t)$, from (54) we get (49), i.e.,

$$
U_{\mathrm{av}}(t)=\frac{c}{s+c}\left\{-k\left[\vartheta_{\mathrm{av}}(t)+\int_{0}^{D} u_{\mathrm{av}}(\sigma, t) d \sigma\right]\right\} \text {. }
$$

Let us now consider $w(D, t)$. It is easily seen that

$$
w_{t}(D, t)=\partial_{t} u_{\mathrm{av}}(D, t)+k u_{\mathrm{av}}(D, t),
$$

where $\partial_{t} u_{\mathrm{av}}(D, t)=\dot{U}_{\mathrm{av}}(t)$. The inverse of (51) is given by $u_{\text {av }}(x, t)=w(x, t)-k\left[e^{-k x} \tilde{\vartheta}_{\mathrm{av}}(t)+\int_{0}^{x} e^{-k(x-\sigma)} w(\sigma, t) d \sigma\right]$.

Plugging (54) and (57) into (56), we get

$$
\begin{aligned}
w_{t}(D, t)= & -c w(D, t)+k w(D, t) \\
& -k^{2}\left[e^{-k D} \tilde{\vartheta}_{\mathrm{av}}(t)+\int_{0}^{D} e^{-k(D-\sigma)} w(\sigma, t) d \sigma\right] .
\end{aligned}
$$

\section{E. Lyapunov-Krasovskii Functional}

Now consider the following Lyapunov functional

$$
V(t)=\frac{\tilde{\vartheta}_{\mathrm{av}}^{2}(t)}{2}+\frac{a}{2} \int_{0}^{D}(1+x) w^{2}(x, t) d x+\frac{1}{2} w^{2}(D, t),
$$

where the parameter $a=1 / k$. As computed in [3], one gets from (58)

$$
\begin{aligned}
\dot{V}(t) \leq & -\frac{1}{4 a} \tilde{\vartheta}_{\mathrm{av}}^{2}(t)-\frac{a}{4(1+D)} \int_{0}^{D}(1+x) w^{2}(x, t) d x \\
& -\left(c-c^{*}\right) w^{2}(D, t)
\end{aligned}
$$

where $c^{*}=\frac{a(1+D)}{2}+k+a\left|k^{2} e^{-k D}\right|^{2}+\frac{1}{a}\left\|k^{2} e^{-k(D-\sigma)}\right\|^{2}$. Hence, from (59), if $c$ is chosen such that $c>c^{*}$, we obtain

$$
\dot{V}(t) \leq-\mu V(t)
$$

for some $\mu>0$. Thus, the closed-loop system is exponentially stable in the sense of the full state norm

$$
\left(\left|\tilde{\vartheta}_{\mathrm{av}}(t)\right|^{2}+\int_{0}^{D} w^{2}(x, t) d x+w^{2}(D, t)\right)^{1 / 2}
$$

i.e., in the transformed variable $\left(\tilde{\vartheta}_{\mathrm{av}}, w\right)$.

\section{F. Exponential Stability Estimate (in $L_{2}$ norm) for the Average System (47)-(49)}

To obtain exponential stability in the sense of the norm $\left(\left|\tilde{\vartheta}_{\mathrm{av}}(t)\right|^{2}+\int_{0}^{D} u_{\mathrm{av}}^{2}(x, t) d x+u_{\mathrm{av}}^{2}(D, t)\right)^{1 / 2}$, we need to show there exist positive numbers $\alpha_{1}$ and $\alpha_{2}$ such that

$$
\alpha_{1} \Psi(t) \leq V(t) \leq \alpha_{2} \Psi(t)
$$

where $\Psi(t) \triangleq\left|\tilde{\vartheta}_{\mathrm{av}}(t)\right|^{2}+\int_{0}^{D} u_{\mathrm{av}}^{2}(x, t) d x+u_{\mathrm{av}}^{2}(D, t)$, or equivalently

$$
\Psi(t) \triangleq\left|\tilde{\theta}_{a v}(t-D)\right|^{2}+\int_{t-D}^{t} U_{\mathrm{av}}^{2}(\tau) d \tau+U_{\mathrm{av}}^{2}(t)
$$

This is straightforward to establish by using (51), (57), (58) and employing the Cauchy-Schwartz inequality and other calculations, as in the proof of Theorem 2.1 in [8]. Hence, with (60), we get

$$
\Psi(t) \leq \frac{\alpha_{2}}{\alpha_{1}} e^{-\mu t} \Psi(0)
$$

which completes the proof of exponential stability.

\section{G. Invoking Averaging Theorem}

Using (6), (10) and (11), the dynamics of the estimation error of the Hessian can be written as

$$
\dot{\tilde{\gamma}}=\omega_{r}\left[\tilde{\gamma}+H^{-1}\right]\left[1-h^{(n+2)}\left(\tilde{\gamma}+H^{-1}\right)\right] .
$$

Now, note that the closed-loop system (5), (30) and (65) can be rewritten as:

$$
\begin{aligned}
\dot{\tilde{\theta}}(t-D) & =U(t-D), \\
\dot{U}(t) & =-c U(t)-c k\left[z(t)+\int_{t-D}^{t} U(\tau) d \tau\right], \\
\dot{\tilde{\gamma}}(t) & =\omega_{r}\left[\tilde{\gamma}(t)+H^{-1}\right] \\
& \times\left[1-h^{(n+2)}\left(\tilde{\gamma}(t)+H^{-1}\right)\right] .
\end{aligned}
$$

where $\xi(t)=[\tilde{\theta}(t-D), U(t), \tilde{\gamma}(t)]^{T}$ is the state vector. Moreover, from (7) and (9), one has

$$
\dot{\xi}(t)=f\left(\omega t, \xi_{t}\right),
$$

where $\xi_{t}(\Theta)=\xi(t+\Theta)$ for $-D \leq \Theta \leq 0$ and $f$ is an appropriate continuous functional, such that the averaging theorem by [9], [10] can be applied considering $\omega=1 / \epsilon$. We conclude that the equilibrium $\tilde{\gamma}_{\text {av }}(t)=0$ of the linearized error system (50) is exponentially stable since $k_{r}>0$.

In addition, from (64), the origin of the average closedloop system (47)-(49) with transport PDE for delay representation is also exponentially stable. Thus, there exist positive constants $\alpha$ and $\beta$ such that all solutions satisfy $\Upsilon(t) \leq \alpha e^{-\beta t} \Upsilon(0), \forall t \geq 0$, where $\Upsilon(t) \triangleq\left|\tilde{\gamma}_{\text {av }}(t)\right|^{2}+$ $\left|\tilde{\theta}_{\mathrm{av}}(t-D)\right|^{2}+U_{\mathrm{av}}^{2}(t)+\int_{t-D}^{t} U_{\mathrm{av}}^{2}(\tau) d \tau$.

Then, according to the averaging theorem [9], [10], for $\omega$ sufficiently large, (66)-(68) has a unique locally exponentially stable periodic solution around the origin satisfying (31). See [3] for computation of (32).

\section{Simulation Results}

Consider the following static cubic nonlinear map

$$
h(\theta)=-(\theta-0.5)^{3}+\theta,
$$

which is subject to output delay $D=5 \mathrm{~s}$ where we maximize $h(\hat{\theta})$ according to (2). However, recall that $y=h(\theta(t-D))$. Hence, we will plot the variable $\theta$ instead of $\hat{\theta}$. Fig. 2 shows (70) and its first derivative.

We use low-pass and washout filters with corner frequencies $\left(\omega_{h}\right.$ and $\left.\omega_{l}\right)$ to improve the controller performance (see [11, Figure 4]). We present simulations of the predictor (30), where $c=20, z$ is given by (9) with $\widehat{h^{(j)}}$ as in (7) and $\gamma$ in (6). The tests are performed with the $\omega_{h}=\omega_{l}=1$. Fig. 2 shows maximization of the first derivative of (70), where after the transient, the Riccati equation converges close to the actual value of the Hessian's inverse. The initial transient in $\gamma$ after the controller "kicks in" at $D=5 \mathrm{sec}$, is driven by 


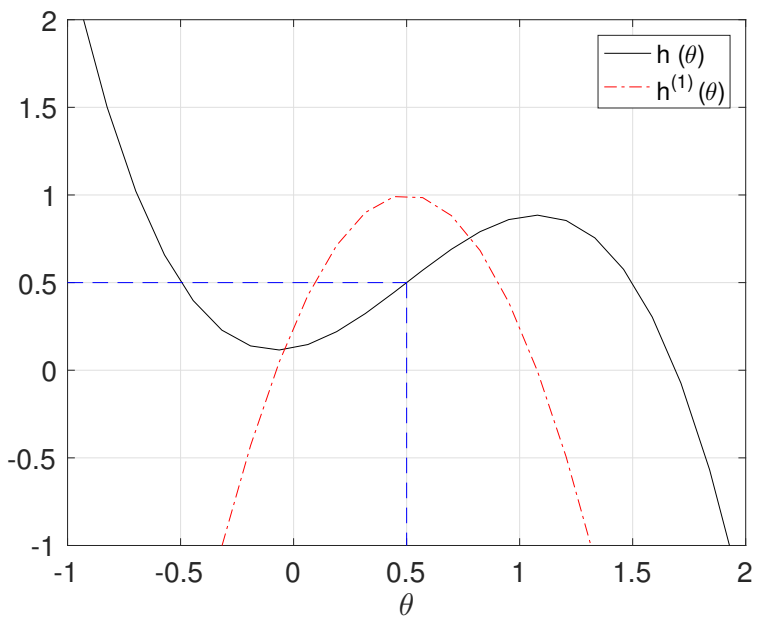

Fig. 2. The derivatives of $h^{(n)}(\theta)$ for $\mathrm{n}=\left[\begin{array}{ll}0, & 1]\end{array}\right.$ where $h^{(0)}(\theta)=h(\theta)=$ black (continuous), $h^{(1)}(\theta)=$ red (semicolon).

$h^{(2)}<0$ and makes $\gamma$ increasingly positive. The output $y(t)$ converges to the value where the desired derivative has its extremum.

\section{Conclusions}

In this work, we present and prove local stability of a Newton-based extremum seeking algorithm in presence of input-output delays, which maximizes arbitrary $n^{\text {th }}$ derivative of an unknown static map. The only available measurements are from the map itself and not of its derivatives. We give a rigorous proof in terms of backstepping transformation and averaging analysis in infinite dimensions for locally quadratic objective functions. The delay is assumed to be known and constant. Generalization to include unknown and time-varying delays as well as stochastic perturbations will be studied in future works.

\section{REFERENCES}

[1] T. R. Oliveira, M. Krstić and D. Tsubakino, "Multiparameter Extremum Seeking with Output Delays", American Control Conference, pp. 152-158, 2015.

[2] T. R. Oliveira, M. Krstić and D. Tsubakino, "Extremum Seeking for Static Maps with Delays", IEEE Transactions on Automatic Control, 2016, doi: 10.1109/tac.2016.2564958.

[3] T. R. Oliveira and M. Krstić, "Newton-based Extremum Seeking under actuator and sensor delays", $12^{\text {th }}$ IFAC Workshop on Time Delay, pp. 304-309, Ann Arbor, 2015

[4] M. Krstić and H. H. Wang, "Stability of extremum seeking feedback for general nonlinear dynamic systems", Automatica, vol. 36, no. 4, pp. 595-601, 2000.

[5] K. Vinther, H. Rasmussen, R. Izadi-Zamanabadi and J. Stoustrup, "Single temperature sensor superheat control using a novel maximum slope-seeking method", International Journal of Refrigeration, vol. 36, no. 3, pp. 1118-1129, 2013.

[6] H. K. Khalil, Nonlinear Systems: Third Edition, Prentice Hall, Jan. 2002.

[7] M. Krstić, Extremum seeking control, in T. Samad and J. Baillieul, Encyclopedia of Systems and Control, Springer, 2014.

[8] M. Krstić, Delay Compensation for Nonlinear, Adaptive and PDE Systems, Birkhäuser, 2009.

[9] J. K. Hale and S. M. V. Lunel, "Averaging in infinite dimensions", Journal of Integral Equations and Applications, vol. 2, pp. 463-494, 1990.
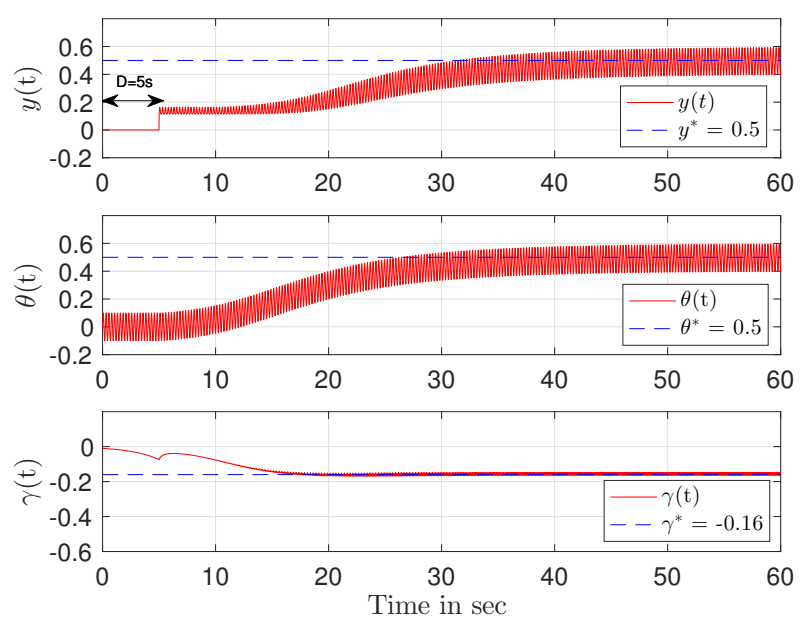

Fig. 3. Maximization of $h^{(1)}(\theta)$ where $h(\theta)=-(\theta-0.5)^{3}+\theta$. The top plot represents $y(t)$, the middle $\theta$, the bottom $\gamma$ and the horizontal axis the time in seconds. For the simulation we use $\omega=25 \mathrm{rad} / \mathrm{sec}, a=0.1$, $D=5 \mathrm{sec}, k=0.1, k_{R}=0.6$. The initial conditions are $\theta_{0}=0$ and $\gamma_{0}=-0.01$. The blue dashed lines represent the targets $y^{*}=0.5$, $\theta^{*}=0.5$ and $\gamma^{*}=-0.16$.

[10] B. Lehman, "The influence of delays when avergaing slow and fast oscillating systems: overview", IMA Journal of Mathematical Control and Information, vol. 19, pp. 201-215, 2002.

[11] A. Ghaffari, M. Krstić and D. Nesić, "Multivariable Newton-based extremum seeking", Automatica, vol. 48, no. 8, pp. 1759-1767, 2012.

[12] G. Mills and M. Krstić, "Maximizing Higher Derivatives of Unknown Maps with Extremum Seeking", IEEE Conference on Decision and Control, pp. 5648-5653, Osaka, 2015.

[13] K. Ariyur and M. Krstić, Real Time Optimization by Extremum Seeking Control, Wiley, 2003.

[14] S. Z. Khong, D. Nesić, Y. Tan and C. Manzie, Unified frameworks for sampled-data extremum seeking control: Global optimisation and multi-unit systems, Automatica, vol. 49, no. 9, pp. 2720-2733, 2013.

[15] Y. Tan, D. Nesić and I. Mareels, On the choice of dither in extremum seeking systems: A case study, Automatica, vol. 44, no. 5, pp. 14461450, 2008.

[16] Y. Tan, D. Nesić and I. Mareels, On non-local stability properties of extremum seeking control, Automatica, vol. 42, no. 6, pp. 889-903, 2006.

[17] D. Dochain, M. Perrier and M. Guay, Extremum seeking control and its application to process and reaction systems: A survey, Mathematics and Computers in Simulation, $6^{\text {th }}$ Vienna International Conference on Mathematical Modelling, vol. 82, no. 3, pp. 369-380, 2011.

[18] D. DeHaan and M. Guay, Extremum-seeking control of stateconstrained nonlinear systems, Automatica, vol. 41, no. 9, pp. 1567-1574, 2005.

[19] M. Guay, S. Dhaliwal and D. Dochain, A time-varying extremumseeking control approach, American Control Conference (ACC), pp. 2643-2648, 2013.

[20] B. Ren, P. Frihauf, R. J. Rafac and M. Krstić, Laser pulse shaping via extremum seeking, Control Engineering Practice, vol. 20, no. 7, pp. 674-683, 2012.

[21] H.-H. Wang, S. Yeung and M. Krstić, Experimental application of extremum seeking on an axial-flow compressor, IEEE Transactions on Control Systems Technology, vol. 8, pp. 300-309, 1999.

[22] P. Frihauf, M. Krstić and T. Basar, Finite-horizon $L Q$ control for unknown discrete-time linear systems via extremum seeking, European Journal of Control, vol. 19, pp. 399-407, 2013.

[23] D. Nešić, Extremum Seeking Control: Convergence Analysis, European Journal of Control, vol. 15, pp. 331-347, 2009.

[24] M. Titica, D. Dochain and M. Guay, Adaptive Extremum Seeking Control of Fed-Batch Bioreactors, European Journal of Control, vol. 9, pp. 618-631, 2003. 